\title{
A SIMPLE DISTRIBUTED RGC MODEL OF MOSFET FOR PRE-PINCH OFF REGION
}

\author{
UMESH KUMAR and ARUN MIRCHANDANI \\ Department of Electrical Engineering, Indian Institute of Technology, Hauz Khas, \\ New Delhi-11016, India.
}

(Received March 22, 1986; in final form July 25, 1986)

The differential equation describing the small signal behavior of a MOSFET channel is derived. Based on the analogy of the channel to distributed transmission lines, which has been very well established in literature, an entirely new RGC line model of MOSFET is presented. The element values of the line are determined by equivalence to a general distributed transmission line and subsequently the model is lumped into a single section in two possible $\Pi$ and $T$ representations. The postulated model considerably simplifies the study of the properties and behavior of MOSFET structures and can be suitably utilized in analysis and Computer Aided Design.

\section{INTRODUCTION}

The transmission line approach to model microelectronic circuit components has been commonly used to evaluate transient and frequency responses. This theory has been applied to a considerably large number of cases for thin film and diffusion resistors, capacitors and conductors and the undesirable interaction between different components of integrated circuits. The transmission lines have also been successfully used in simulation of active microelectronic elements; in particular, the field effect devices. Also, any integrated circuit employing FETs may be considered as consisting of transmission lines and additional lumped elements.

There have been numerous attempts to derive a distributed equivalent circuit model. These include those by Hoffmann ${ }^{1,2}$, Kocprgak $^{3}$, McNutt et $\mathrm{al}^{4}$, Popor \& Bickart ${ }^{5}$ and Lonngren ${ }^{6}$. A great deal of work on this topic has been reported by Barsan ${ }^{7-14}$ who has made the extrapolation to charge transfer devices ${ }^{15}$. We have proceeded from an entirely different method to arrive at such a representation. 


\section{THEORETICAL ANALYSIS AND DERIVATION}

Fig. 1 shows an n-channel MOSFET which the source and substrate short circuited to the ground. A voltage Vds consisting of a d.c. bias Vds is applied between the drain and the source. $I_{D}$ is the output drain current.

Now if

Eox: permittivity of the oxide

tox: oxide thickness

$\mathrm{Z}$ : is the MOSFET width in the direction transverse to the current flow

$\mathrm{U}$ : total gate channel potential at any point $\mathrm{x}$ in the channel.

$\mathrm{C}_{\mathrm{ox}}=\frac{\epsilon_{\mathrm{ox}} \cdot \mathrm{Z}}{\mathrm{t}_{\mathrm{ox}}}$

gate channel capacitance per unit length of the channel. Then

$\mathrm{C}_{\mathrm{ox}} \cdot \mathrm{U}=$ mobile channel charge per unit length of the channel.

Now if

$\mu=$ mobility of the carriers (electrons) in the channel,

then

$\mu \frac{\partial u}{\partial x}=$ velocity of the carries in the channel.

Hence

$\left(\mathrm{C}_{\mathrm{ox}} \cdot \mathrm{U}\right)\left(\mu \frac{\partial \mathrm{u}}{\partial \mathrm{x}}\right)=\mathrm{I}$

channel current at any point $\mathrm{x}$ in the channel.

Assuming that we can express the gate potential and current $I$ as the sum of a.c. and d.c. components, i.e.,

$U(x, t)=v(x)+u(x) e^{j \omega t}$ 


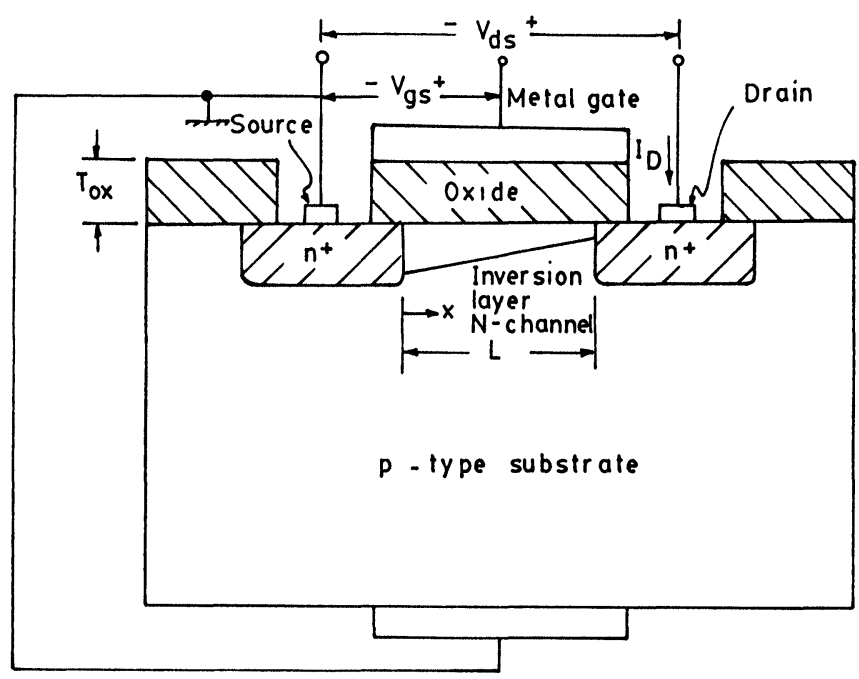

FIGURE 1 General MOSFET structure.

and

$I(x, t)=I_{o}(x)+i(x) e^{j \omega t}$

substituting in equation (1) we obtain

$$
\begin{aligned}
I= & \mu C_{o x}\left[v(x)+u(x) e^{j \omega t}\right]\left[\frac{d v(x)}{d x}+\frac{d u(x)}{d x} \cdot e^{j \omega t}\right] \\
= & \mu C_{o x}\left[v(x) \cdot \frac{d v(x)}{d x}+u(x) \frac{d v(x)}{d x} \cdot e^{j \omega t}\right. \\
& +v(x) \cdot \frac{d u(x)}{d x} \cdot e^{j \omega t}+u(x) \cdot \underbrace{\left.\frac{d u(x)}{d x} \cdot e^{2 j \omega t}\right]}_{\text {ignored }}
\end{aligned}
$$

Ignoring the second order term in the a.c. component under small signal approximation and equating the time independent and time dependent terms on both the sides we get 
$I_{o}(x)=\mu C_{o x} v(x) \cdot \frac{d v(x)}{d x}$

and

$$
\mathrm{i}(\mathrm{x})=\mu \mathrm{C}_{\mathrm{ox}} \frac{\mathrm{d}}{\mathrm{dx}}(\mathrm{u}(\mathrm{x}) \cdot \mathrm{v}(\mathrm{x}))
$$

Now considering an incrementat section of length $\Delta \mathrm{x}$ of the channel we can write

$$
\begin{aligned}
\Delta \mathrm{I} & =\frac{\partial}{\partial \mathrm{t}}[\mathrm{Q} \Delta \mathrm{x}] \\
& =\frac{\partial}{\partial \mathrm{t}}\left[\mathrm{C}_{\mathrm{ox}} \cdot \mathrm{U} \cdot \Delta \mathrm{x}\right]
\end{aligned}
$$

where Q: channel charge per unit length

or

$\frac{\partial I}{\partial x}=\frac{\partial}{\partial t}\left[C_{o x} \cdot U\right]$

Again, substituting for $U+I$ from equations (2) $+(3)$ in (6) we get

$\frac{\partial}{\partial x}\left[I_{o}+i(x) \cdot e^{j \omega t}\right]=\frac{\partial}{\partial t}\left\{C_{o x}\left[v(x)+u(x) e^{j \omega t}\right]\right\}$

or

$\frac{d I_{o}}{d x}+\frac{d i(x)}{d x} \cdot e^{j \omega t}=\frac{\partial}{\partial t}\left[C_{o x} \cdot v(x)\right]+\frac{\partial}{\partial t}\left[C_{o x} \cdot u(x) \cdot e^{j \omega t}\right]$

or

$\frac{\operatorname{di}(x)}{d x} \cdot e^{j \omega t}=\frac{\partial}{\partial t}\left[C_{o x} \cdot u(x) \cdot e^{j \omega t}\right]$

because $C_{o x} \cdot v(x)$ is time independent.

Thus 
$\frac{\mathrm{di}(\mathrm{x})}{\mathrm{dx}}=\mathrm{C}_{\mathrm{ox}} \cdot \mathrm{u}(\mathrm{x}) \cdot \mathrm{j} \omega$

From (5) we have

$$
\begin{aligned}
\mathrm{i}(\mathrm{x}) & =\mu \mathrm{C}_{\mathrm{ox}} \frac{\mathrm{d}}{\mathrm{dx}}[\mathrm{v}(\mathrm{x}) \cdot \mathrm{u}(\mathrm{x})] \\
& =\mu \mathrm{C}_{\mathrm{ox}}\left[\mathrm{u}(\mathrm{x}) \cdot \frac{\mathrm{dv}(\mathrm{x})}{\mathrm{dx}}+\mathrm{v}(\mathrm{x}) \cdot \frac{\mathrm{du}(\mathrm{x})}{\mathrm{dx}}\right]
\end{aligned}
$$

Differentiating we get

$\frac{\mathrm{di}(\mathrm{x})}{\mathrm{dx}}=\mu \mathrm{C}_{\mathrm{ox}}\left[\mathrm{u}(\mathrm{x}) \frac{\mathrm{d}^{2}}{\mathrm{dx}^{2}} \mathrm{v}(\mathrm{x})+2 \frac{\mathrm{d}}{\mathrm{dx}} \mathrm{u}(\mathrm{x}) \cdot \frac{\mathrm{d}}{\mathrm{dx}} \mathrm{v}(\mathrm{x})+\mathrm{v} \frac{\mathrm{d}^{2}}{\mathrm{dx}^{2}} \mathrm{u}(\mathrm{x})\right]$

comparing with equation (7) we get

$V(x) \frac{d^{2}}{d x^{2}} u(x)+2 \frac{d}{d x} u(x) \cdot \frac{d}{d x} v(x)+u(x) \cdot \frac{d^{2}}{d x^{2}} v(x)=\frac{j \omega u}{\mu}$

or

$\frac{d^{2} u(x)}{d x^{2}}+\left(\frac{2}{v(x)} \cdot \frac{d v(x)}{d x}\right) \frac{d u(x)}{d x}+\left(\frac{1}{v(x)} \cdot \frac{d^{2} v(x)}{d x^{2}}-\frac{j \omega}{\mu v}\right)=0$

which can be written as

$u^{\prime \prime}+\left(\frac{2}{v} \cdot v^{\prime}\right) \cdot u^{\prime}+\left(\frac{1}{v} \cdot v^{\prime \prime}-\frac{j \omega}{\mu v}\right) u=0$

where a "'” denotes a derivative v.r.t. $x$. This is the standard differential equation controlling the operation of MOS transistor.

Now for the general transmission line structure shown in Fig. 2,

$\mathrm{Z}$ : Series impedence per unit length

Y: Shunt admittance per unit length

we can write, 


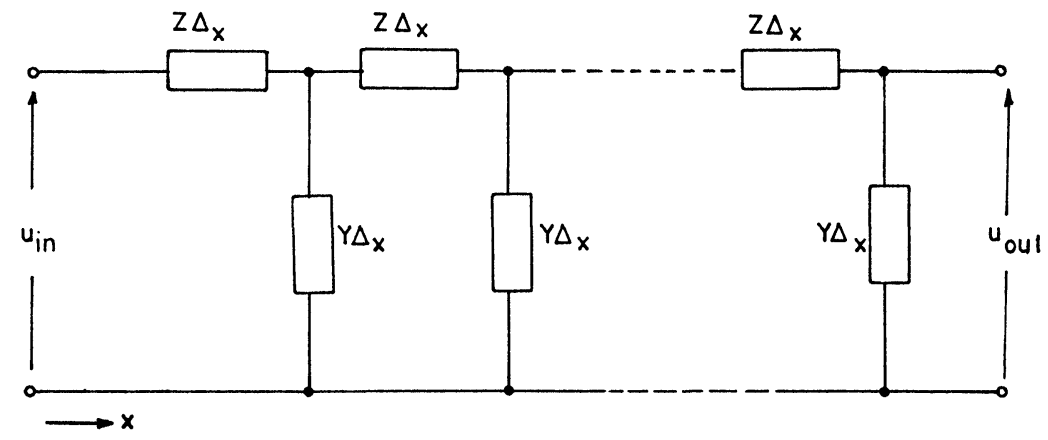

FIGURE 2 General transmission line.

$u^{\prime \prime}-\frac{Z^{\prime}}{Z} u-Y Z u=0$

as the characteristic equation where $u(x)$ is the small signal line voltage at any point $x$.

Equation (9) is exactly analogous to equation (8) obtained for the MOS channel. Hence by comparison we get,

$\frac{\mathrm{Z}^{\prime}}{\mathrm{Z}}=-\mathrm{Z} \frac{\mathrm{v}^{\prime}}{\mathrm{v}}$

and,

$\frac{\mathrm{v}^{\prime \prime}}{\mathrm{v}}-\frac{\mathrm{j} \omega}{\mu \mathrm{v}}=-\mathrm{YZ}$

from (10) we get upon integrating

$\ln Z=-2 \ln v+\ln \mathrm{k}$

or

$\mathrm{Z}=\mathrm{k} / \mathrm{v}^{2}$ 
which is purely real and hence a pure series of resistances, $r$, per unit length.

Also from (11) $\mathrm{Y}$ can be expressed as

$$
\begin{aligned}
Y & =\frac{j \omega}{\omega v Z}-\frac{v^{\prime \prime}}{v Z} \\
& =\frac{j \omega v}{k \mu}-\frac{v^{\prime \prime}}{v Z} \\
& =\frac{j \omega v}{k \mu}-\frac{v^{\prime \prime} v}{k} \\
& =g+j \omega c
\end{aligned}
$$

where

$\mathrm{g} \equiv-\frac{\mathrm{v} " \mathrm{v}}{\mathrm{k}}$

as the shunt conductance per unit length and

$\mathrm{c} \equiv \frac{\mathrm{v}}{\mathrm{k} \mu}$

as the shunt capacitance per unit length.

\section{PROPOSED MODEL FOR MOSFET}

Hence, the resulting small signal distributed RGC line model of the MOSFET intrinsic portion can be represented as shown in Fig. 3.

To determine the values of the elements as functions of distance, we need to know the voltage distribution $v$ as a function of $x$.

Since the d.c. current at any point $\mathrm{x}$ is given by

$$
\mathrm{I}^{\mathrm{o}}=\mu \mathrm{C}_{\mathrm{ox}} \mathrm{v}(\mathrm{x}) \cdot \frac{\partial \mathrm{v}}{\partial \mathrm{x}}
$$

and is constant throughout the channel independent of $\mathbf{x}$, we have: 


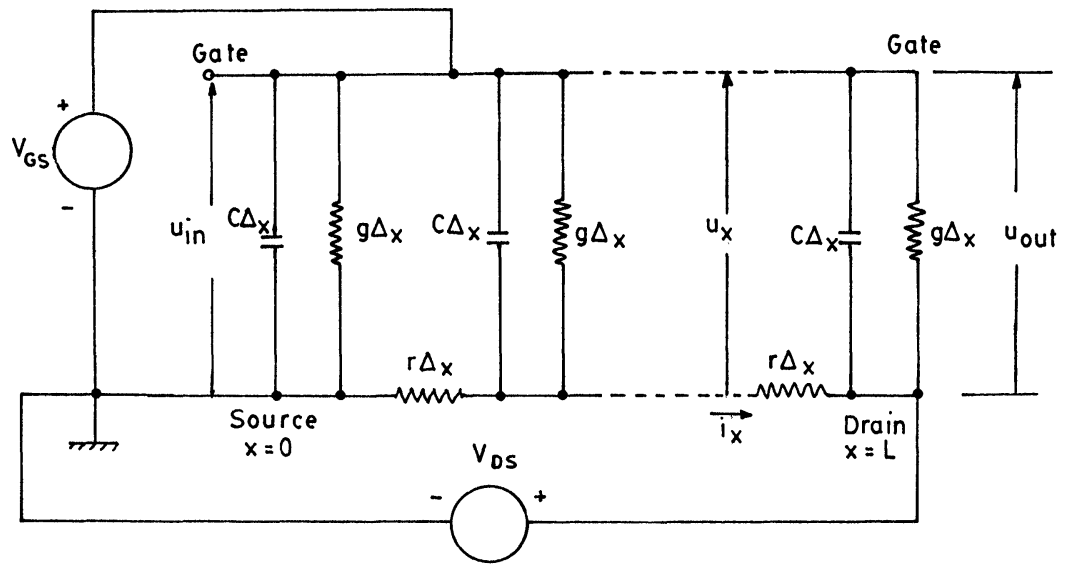

FIGURE 3 Proposed distributed RGC line model of MOSFETs.

$\mathrm{V} \cdot \frac{\mathrm{dv}(\mathrm{x})}{\mathrm{dx}}=$ constant

Integrating both sides from o to $\mathrm{x}$ we get

$\mathrm{v}(\mathrm{x})=\mathrm{c}_{1} \mathrm{x}+\mathrm{c}_{2}$

Apply the boundry conditions

$\mathrm{v}(\mathrm{o})=\mathrm{V}_{\mathrm{g}}-\mathrm{V}_{\mathrm{T}}$

and

$\mathrm{v}(\mathrm{L})=\mathrm{V}_{\mathrm{g}}-\mathrm{V}_{\mathrm{D}}-\mathrm{V}_{\mathrm{T}}$

we get

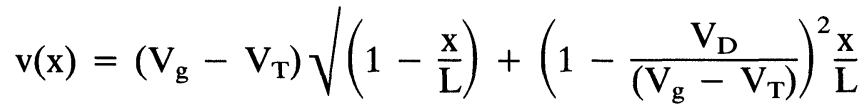

Now we have 
$\mathrm{r}=\frac{\mathrm{k}}{\mathrm{v}_{2}}$

Therefore the total resistance of the channel is

$$
\begin{aligned}
R & =k \int_{V_{g}-V_{T}}^{V_{g}-V_{T}-V_{D}} \frac{1}{v^{2}(x)} \cdot d x \\
& =\frac{k V_{D}}{\left(V_{g}-V_{D}\right)\left[V_{D}-\left(V_{g}-V_{T}\right)\right]}
\end{aligned}
$$

Similarly
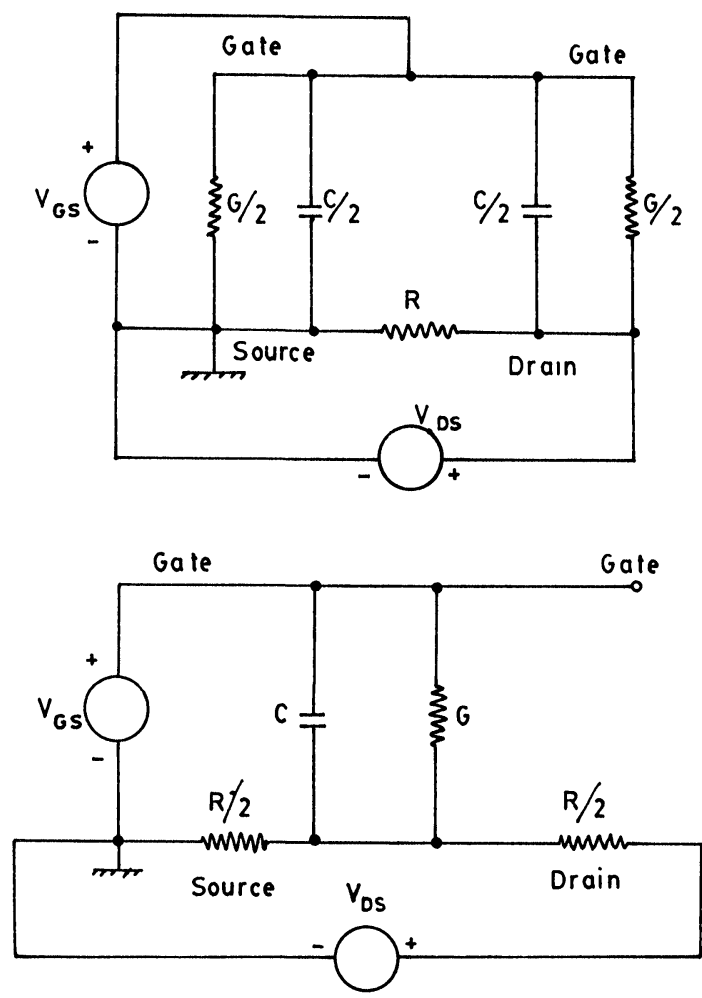

FIGURE 4 Single section models of MOSFET. 
$\mathrm{C}=\frac{1}{\mathrm{k} \mu} \int_{\mathrm{V}_{\mathrm{g}}-\mathrm{V}_{\mathrm{T}}}^{\mathrm{V}_{\mathrm{g}}-\mathrm{V}_{\mathrm{T}}-\mathrm{V}_{\mathrm{D}}} \mathrm{V} \cdot \mathrm{dv}$

gives the total capacitance as

$C=\frac{V_{D}\left[V_{D}-2\left(V_{g}-V_{t}\right)\right]}{2 k \mu}$

and the total shunt conductance is

$$
\begin{aligned}
G= & \frac{V_{D 2}\left[V_{D}-2\left(V_{g}-V_{T}\right)\right]^{2}}{4 k L^{2}} \times\left\{\left(V_{G}-V_{T}\right)\left[\ln \left(V_{G}-V_{T}\right)-1\right]\right. \\
& -\left(V_{G}-V_{T}-V_{D}\right)\left[\ln \left(V_{G}-V_{T}-V_{D}-1\right)\right]
\end{aligned}
$$

we can lump the distributed model derived above and obtain single $\mathrm{T}$ or $\Pi$ analog model which can be represented as shown in Fig. 4.

\section{REFERENCES}

1. K. Hoffmann, "MOS transmission line and its equivalent circuit model," Siemens Forsch. -u. Entarickl - Ber, Vol. 5, pp. 257-261. 1976.

2. K. Hoffmann, "Surface change transport with MOS transmission line", Solid State Electron, Vol. 20, pp. 177-181, 1977.

3. T. Kocprzak, "The linear MOS transistor model taking into account the frequency dispersion of the MTS structure capacitance effect", Arch. Elektro tech., Vol. 26, no. 1, pp. 156-177, 1977.

4. M.J. McNutt, J.M. Holt Ramp and C.T. Sah, "Differential equation solutions of MOS transmission line models generalized to lossy cases", solid state electronics, Vol. 21, pp. 1145-1148, 1978.

5. V.P. Popov and T.A. Bickart, "RC transmission line with nonlinear resistance: Large signal response computation”, IEEE Trans, Vol. CAS-21, no. 5, pp. 666671, Sept. 1979.

6. K.E. Lonngren, "Ananalytical solution of the nonlinear transport equation that descrines an MOS transmission line", Solid State Electronics, Vol. 21, pp. 481484, 1978.

7. R.M. Barsan, "Operation of a resistive States MOS transmission line with time varying State Voltages and field dependent carrier mobility", Rev. Roum, Phys., Vol. 23, no. 5, pp. 505-510, 1979.

8. R.M. Barsan, "A model for the charge proporgation in an MOS transmission 
lie", Rev. Roum, Phys., Vol. 23, no. 2, pp. 189-195, 1978.

9. R.M. Barsan, "Computer Simulation and distributed model analysis of the free charge transport in an MOS transmission line". Rev. Roun, Phys., Vol. 23, no. 4, pp. 333-351, 1978.

10. R.M. Barsan, "Transient analysis of the state potential for a resistive gate MOS structure in deep depletion,” Rev. Roum. Phys. Vol. 22, no. 9, pp. 959-974, 1977.

11. R.M. Barsan, "Model for charge propagation in a resistive state MOS structure", Electron. left, Vol. 13, no. 16, pp. 475-477, 4 August 1977.

12. R.M. Bansan, "Free charge propagation in a resistive sa safo MOS transmission line T. IEEE Trans. Vol. ED-25, no. 9, pp. 1109-1119, September 1978.

13. R.M. Barsan, "Distributed model computer analysis of the free charge transport in an MOS transmission line", Int. J. Electronics, Vol. 45, no. 1, pp. 17-35, 1928.

14. R.M. Barsan, "Transient behaviour of the gate potential in an MOS transmission line", Int. J. Electronics, Vol. 45, no. 1, pp. 1-16, 1978.

15. R.M. Barsan, "Distributed model analysis of charge - coupled and bucket brogate devices”, Proc. Inst. Elect. Engg. Vol. 123, pp. 505-508, June 1970. 

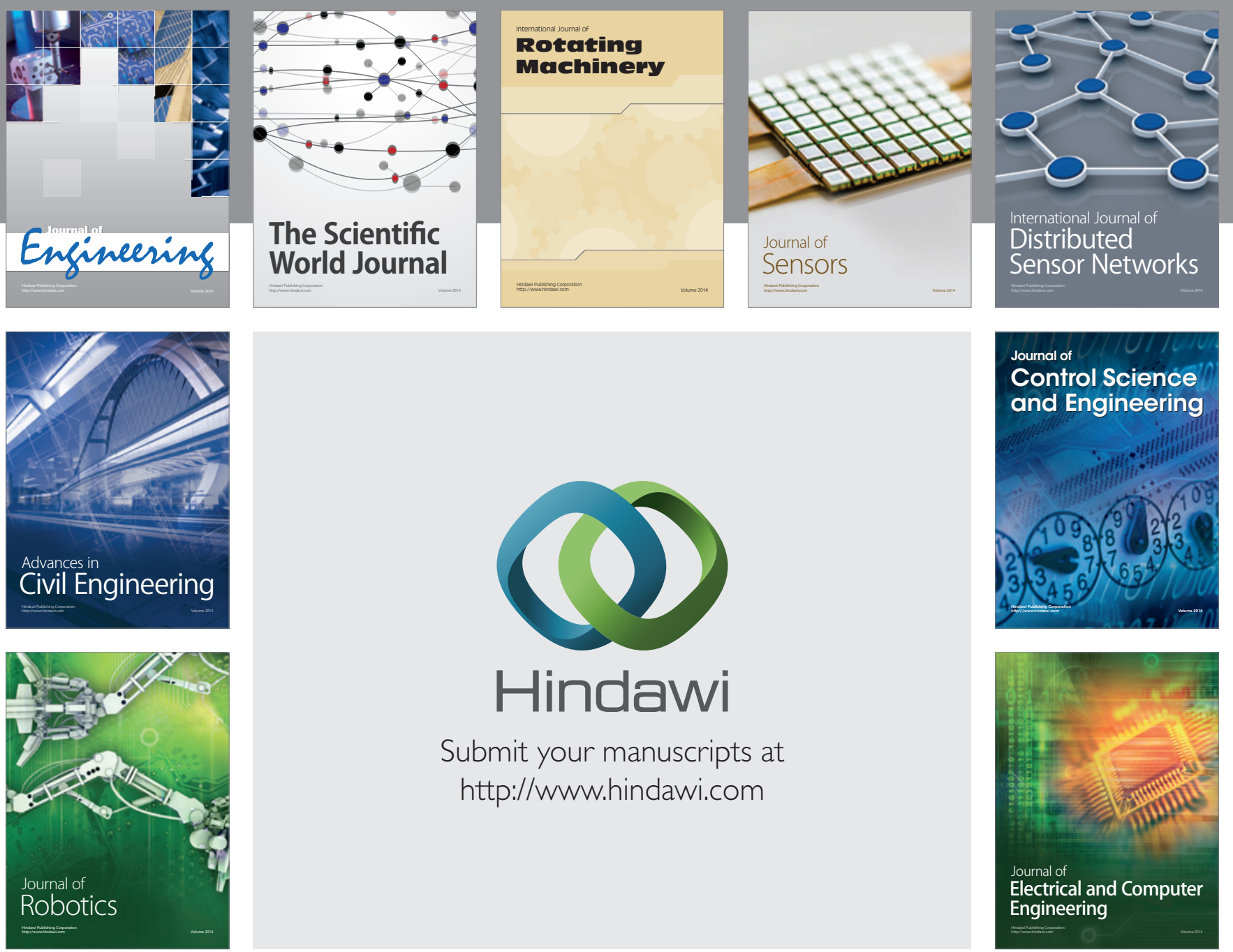

Submit your manuscripts at

http://www.hindawi.com
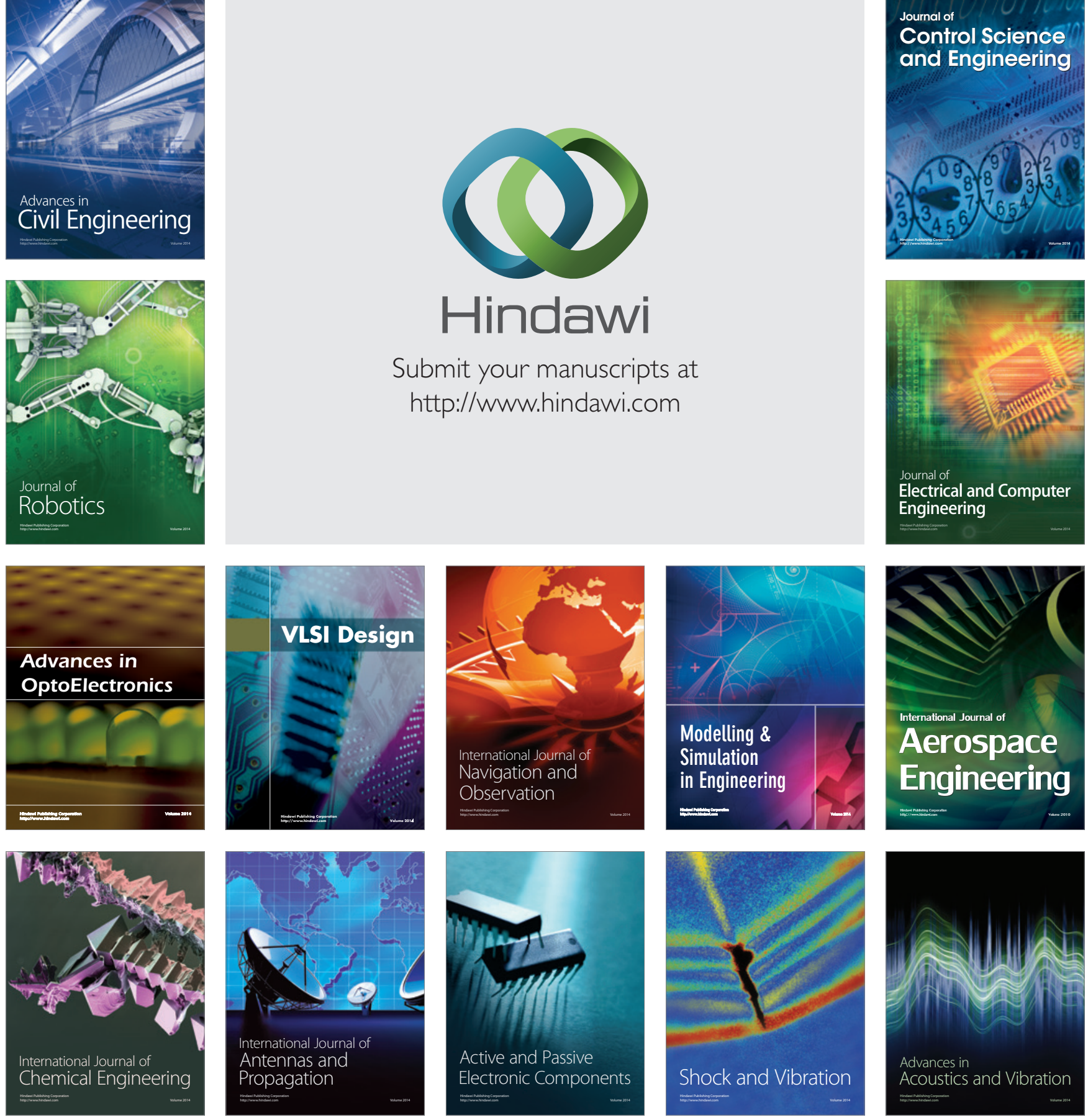